\title{
Association of Serum Uric Acid Levels in Meige's Syndrome
}

\author{
Haochen Guan ${ }^{1 \dagger}$, Zhi Geng ${ }^{2 t}$, Weijie Yuan ${ }^{1 *}$ and Bowen Chang ${ }^{3 *}$ \\ 'Department of Nephrology, Shanghai General Hospital, Shanghai Jiao Tong University School of Medicine, Shanghai, \\ China, ${ }^{2}$ Department of Neurology, Second People's Hospital of Hefei City, The Hefei Affiliated Hospital of Anhui Medical \\ University, Hefei, China, ${ }^{3}$ Division of Life Sciences and Medicine, Department of Neurosurgery, The First Affiliated Hospital \\ of USTC, University of Science and Technology of China, Hefei, China
}

\section{OPEN ACCESS}

Edited by:

Nicholas James Ashton, University of Gothenburg, Sweden

Reviewed by:

Carlos Henrique Ferreira

Camargo,

Federal University of Paraná, Brazi

Paul Matthew D. Pasco,

University of the Philippines Manila, Philippines

Eduardo Joaquim Lopes Alho, University of São Paulo, Brazil

Rubens Gisbert Cury,

University of São Paulo, Brazil

*Correspondence:

Bowen Chang

changbowen21@163.com

Weijie Yuan

ywj4168@163.com

†These authors have contributed equally to this work

Specialty section:

This article was submitted to Neurodegeneration,

a section of the journal

Frontiers in Neuroscience

Received: 07 August 2021 Accepted: 10 September 2021

Published: 01 October 2021

Citation:

Guan H, Geng Z, Yuan W and Chang B (2021) Association of Serum

Uric Acid Levels in Meige's

Syndrome.

Front. Neurosci. 15:755056. doi: 10.3389/fnins.2021.755056
Uric acid (URIC) is a natural antioxidant, and it has been shown that low levels of URIC could be a risk factor for the development of Parkinson's disease. Our aim was to investigate whether URIC also plays a role in Meige's syndrome (MS). We conducted a cohort study to compare serum URIC levels between patients with MS and healthy controls. In addition, we analyzed the impact of URIC on the risk of MS and symptom severity. Compared with normal subjects, URIC content was remarkably decreased in MS patients. In addition, URIC was regarded as a protective factor for MS, as verified by multivariate logistic regression models. We also found non-linear relationships between the levels of serum URIC and the incidence rate of MS and the Burke-Fahn-Marsden dystonia rating scale score. Our study is the first to show a connection between serum URIC levels and MS. Low serum URIC levels indicate an increased risk of MS incidence and more severe clinical symptoms. Our findings provide new insights into the prevention and treatment of MS.

Keywords: Meige's syndrome, uric acid, antioxidants, movement disorders, Burke-Fahn-Marsden dystonia rating scale score

\section{INTRODUCTION}

Meige's syndrome (MS) is a dystonia characterized by bilateral eyelid and involuntary facial muscle movement (Greene et al., 1995; LeDoux, 2009). The incidence is approximately 100 cases per 100,000 people (Defazio et al., 2004). MS is currently considered movement disorder that commonly occurs in elderly female patients (Pandey and Sharma, 2017). Although the pathogenesis of movement disorder such as MS and Parkinson's disease (PD) remains unknown, free radical accumulation (such as reactive oxygen species) and decreased antioxidants within brain tissue may be possible mechanisms (Savitt et al., 2006; Poewe et al., 2017).

Serum uric acid (URIC) has been identified as a natural antioxidant in the human body (Stocker et al., 1987; Becker et al., 1991). Recent meta-analyses and controlled studies suggest that reduced levels of URIC are related to PD (Jesus et al., 2013; Wen et al., 2017). In addition, two prospective articles demonstrated that people showing increased URIC contents may have a lower susceptibility to PD (Davis et al., 1996; Gao et al., 2016) and a slower rate of decline of neurological function (Ascherio et al., 2009). A postmortem study revealed that the URIC content within brain tissue (particularly in the striatal substantia nigra and cortex) was remarkably decreased in PD cases compared with normal controls (Church and Ward, 1994; McFarland et al., 2013). These results suggest that URIC may be related to PD progression, since its reduction within brain tissue indicates antioxidant insufficiency. Whether serum URIC has a similar effect on MS is unknown. 
TABLE 1 | Characteristics of the patients with Meige's syndrome and healthy controls.

\begin{tabular}{|c|c|c|c|}
\hline Variables & Healthy control & Meige's syndrome & $P$-value \\
\hline No. & 133 & 80 & \\
\hline Gender & & & 0.011 \\
\hline Male & 72 (54.1\%) & 29 (36.2\%) & \\
\hline Female & 61 (45.9\%) & $51(63.7 \%)$ & \\
\hline Age & $55.5 \pm 17.9$ & $54.2 \pm 10.1$ & 0.547 \\
\hline Uric acid & $317.2 \pm 71.0$ & $250.5 \pm 66.9$ & $<0.001$ \\
\hline CREA & $65.2 \pm 20.9$ & $62.4 \pm 13.9$ & 0.283 \\
\hline BUN & $5.3 \pm 1.4$ & $5.9 \pm 1.5$ & 0.013 \\
\hline ALB & $42.0 \pm 3.7$ & $42.3 \pm 4.1$ & 0.698 \\
\hline Hypertension & & & 0.109 \\
\hline No & 94 (70.7\%) & 48 (60.0\%) & \\
\hline Yes & 39 (29.3\%) & 32 (40.0\%) & \\
\hline
\end{tabular}

TABLE 2 | Association between each variable and Meige's syndrome.

\begin{tabular}{|c|c|c|c|}
\hline Variables & Statistics & Meige's syndrome OR ( $95 \% \mathrm{Cl})$ & $P$-value \\
\hline Gender & & & 0.011 \\
\hline Male & $101(47.418 \%)$ & 1.0 & \\
\hline Female & 112 (52.582\%) & $2.076(1.175,3.668)$ & \\
\hline Age & $55.047 \pm 15.397$ & $0.994(0.978,1.011)$ & 0.502 \\
\hline Uric acid & $292.129 \pm 76.528$ & $0.985(0.978,0.992)$ & $<0.001$ \\
\hline CREA & $64.120 \pm 18.626$ & $0.991(0.977,1.005)$ & 0.223 \\
\hline BUN & $5.531 \pm 1.492$ & $1.266(1.050,1.526)$ & 0.013 \\
\hline ALB & $42.119 \pm 3.875$ & $1.014(0.942,1.093)$ & 0.706 \\
\hline Hypertension & & & 0.111 \\
\hline No & 142 (66.667\%) & 1.0 & \\
\hline Yes & 71 (33.333\%) & $1.607(0.897,2.877)$ & \\
\hline
\end{tabular}

TABLE 3 | Multivariate regression for effect of serum uric acid levels on Meige's syndrome.

\begin{tabular}{|c|c|c|c|c|c|c|}
\hline \multirow{2}{*}{$\begin{array}{l}\text { Variable } \\
\text { Uric acid }\end{array}$} & \multicolumn{2}{|c|}{ Non-adjusted } & \multicolumn{2}{|c|}{ Model I } & \multicolumn{2}{|c|}{ Model II } \\
\hline & OR $(95 \% \mathrm{Cl})$ & $P$-value & OR (95\% Cl) & $P$-value & OR $(95 \% \mathrm{Cl})$ & $P$-value \\
\hline & $0.985(0.980,0.990)$ & $<0.001$ & $0.985(0.980,0.991)$ & $<0.001$ & $0.984(0.979,0.990)$ & $<0.001$ \\
\hline
\end{tabular}

Model I adjusted for age and gender. Model Il adjusted for age, gender, history of hypertension. Cl, confidence interval; OR, odds ratio.

At present, the etiology and pathogenic mechanisms of MS remain unclear. It has been suggested that diverse environmental and genetic factors, mitochondrial dysfunction, and other factors are related to the oxidative stress (OS) of neurons (Steele et al., 2014). This study focused on investigating the serum URIC content in MS and evaluating its impact on the development of MS.

\section{MATERIALS AND METHODS}

\section{Meige's Syndrome Patients and Normal Subjects}

This retrospective cross-sectional study collected medical records from MS cases diagnosed at the Shanghai General Hospital

Abbreviations: BFMDRS, Burke-Fahn-Marsden dystonia rating scale; MS, Meige’s syndrome; OS, oxidative stress; PD, Parkinson's disease; URIC, uric acid. and Hefei Second People's Hospital between January 2018 and February 2021. Patients meeting the following criteria were included: those with MS, the main manifestations were double blepharospasm, oral and mandibular dystonia, and involuntary movements like facial dystonia; those not receiving treatment such as opioids or non-steroidal anti-inflammatory drugs; those without hematological disorders, hyperpyrexia, concurrent infectious disease, severe heart disease, metabolic disorder, inflammatory disease or medication for inflammatory disease, autoimmune disease, severe liver/kidney disease, other malignancies; and those with sufficient data on the biochemical index of fasting blood. In addition, this study also collected medical records of age- and sex-matched healthy subjects who underwent physical examinations at the same hospital. None of the healthy control subjects took any drugs to raise or lower uric acid. Our study protocols were approved by the Institutional Ethics Committee of the hospital. All participants in this study provided informed consent. 


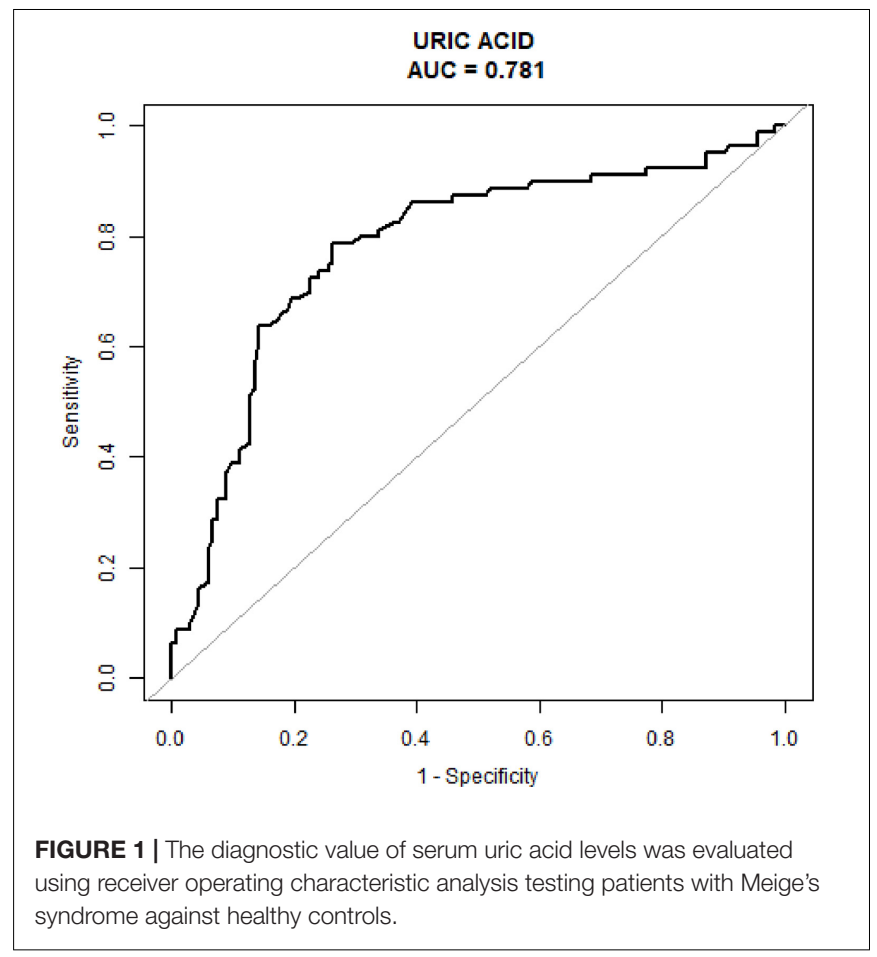

TABLE 4 | The threshold effect of serum uric acid levels on incidence rate of Meige's syndrome assessments.

\begin{tabular}{|c|c|c|}
\hline & OR $(95 \% \mathrm{Cl})$ & $P$-value \\
\hline Uric acid $<337 \mu \mathrm{mol} / \mathrm{L}$ & $0.975(0.968,0.983)$ & $<0.001$ \\
\hline Uric acid $\geq 337 \mu \mathrm{mol} / \mathrm{L}$ & $1.007(0.997,1.018)$ & 0.186 \\
\hline
\end{tabular}

\section{Data Extraction}

Patient clinicopathological and demographic variables, including sex, age, BFMDRS (Burke-Fahn-Marsden dystonia rating scale) score, and history of hypertension were collected from medical records. In addition, blood samples were collected upon admission to conduct kidney and liver tests, which were part of the standard workup. Each specimen was assayed by the Department of Clinical Laboratory $2 \mathrm{~h}$ post-collection. Specifically, URIC, albumin (ALB), blood urea nitrogen (BUN), and creatinine (CREA) levels were examined to assess kidney and liver function. The above clinical variables were determined using standard automatic counters. The epidemiological data, clinical evaluation, and laboratory tests were extracted from the same visit of each patient.

\section{Statistical Analyses}

$\mathrm{R}^{1}$ and Empower $(\mathrm{R})^{2}$ (X\&Y solutions, Inc., Boston, MA, United States) software were used for statistical analysis. First, the normal distribution of variables was assessed using

${ }^{1}$ http://www.R-project.org

${ }^{2}$ www.empowerstats.com the Kolmogorov-Smirnov test, and normally distributed data were assessed by one-way analysis of variance or two-tailed Student's $t$-test. Simultaneously, non-parametric data were compared across diverse groups using the Mann-Whitney $U$ test. A multiple logistic regression model was employed to evaluate the relationships between inflammatory markers and MS. Additionally, the value of the area under the receiver operating characteristic (ROC) curve (AUC) was determined to assess the significance of URIC in the diagnosis of MS. The two-piecewise linear regression model was also utilized to examine the role of URIC in predicting MS and BFMDRS scores using the smoothing function. Typically, trial and error were utilized to determine the threshold level (turning point), such as selecting the turning point down a preset interval and later selecting a turning point giving the maximum model likelihood. After post hoc analysis, we determined the outlier by adopting the value with maximal specificity and sensitivity. Statistical significance was set at $P<0.05$.

\section{RESULTS}

\section{Study Participants}

Altogether, data for $80 \mathrm{MS}$ cases and 133 healthy controls were collected for final analyses. Table 1 shows the demographic data of all study participants. There were $29(36.2 \%)$ male and 51 (63.7\%) female MS patients, with ages ranging between 39 and 81 years. There were $72(54.1 \%)$ male controls, aged between 29 and 86 years.

\section{Comparing the Biochemical Indexes Between Meige's Syndrome Patients and Normal Subjects}

Table 1 shows the features of all study participants. Differences in ALB, BUN, CREA, and diabetes history were not significant between the two groups. In contrast, URIC levels of MS cases $(250.5 \pm 66.9 \mu \mathrm{mol} / \mathrm{L})$ decreased significantly in comparison with healthy controls $(317.2 \pm 71.0 \mu \mathrm{mol} / \mathrm{L}$; $P<0.05)$.

\section{Relationship of Uric Acid With Meige's Syndrome}

Table 2 displays the relationships between MS and diverse variables, such as sex, age, ALB, BUN, CREA, URIC, and hypertension history. URIC content was closely related to MS, and increased URIC content served as a protective factor for MS. Table 3 presents the above relationships assessed through multivariate analyses. As suggested by multivariate analysis, URIC content was closely related to MS (Odds ratio $[\mathrm{OR}]=0.985$; 95\% confidence interval [CI]: 0.980-0.990; $P<0.001)$. After adjusting for confounders such as sex, age, and hypertension history, the results remained largely unchanged, which confirmed that the decreased URIC content served as a risk factor for $\mathrm{CH}(\mathrm{OR}=0.984 ; 95 \% \mathrm{CI}: 0.979-0.990 ; P<0.001)$. The value of URIC in diagnosing MS patients (ROC curves) is 


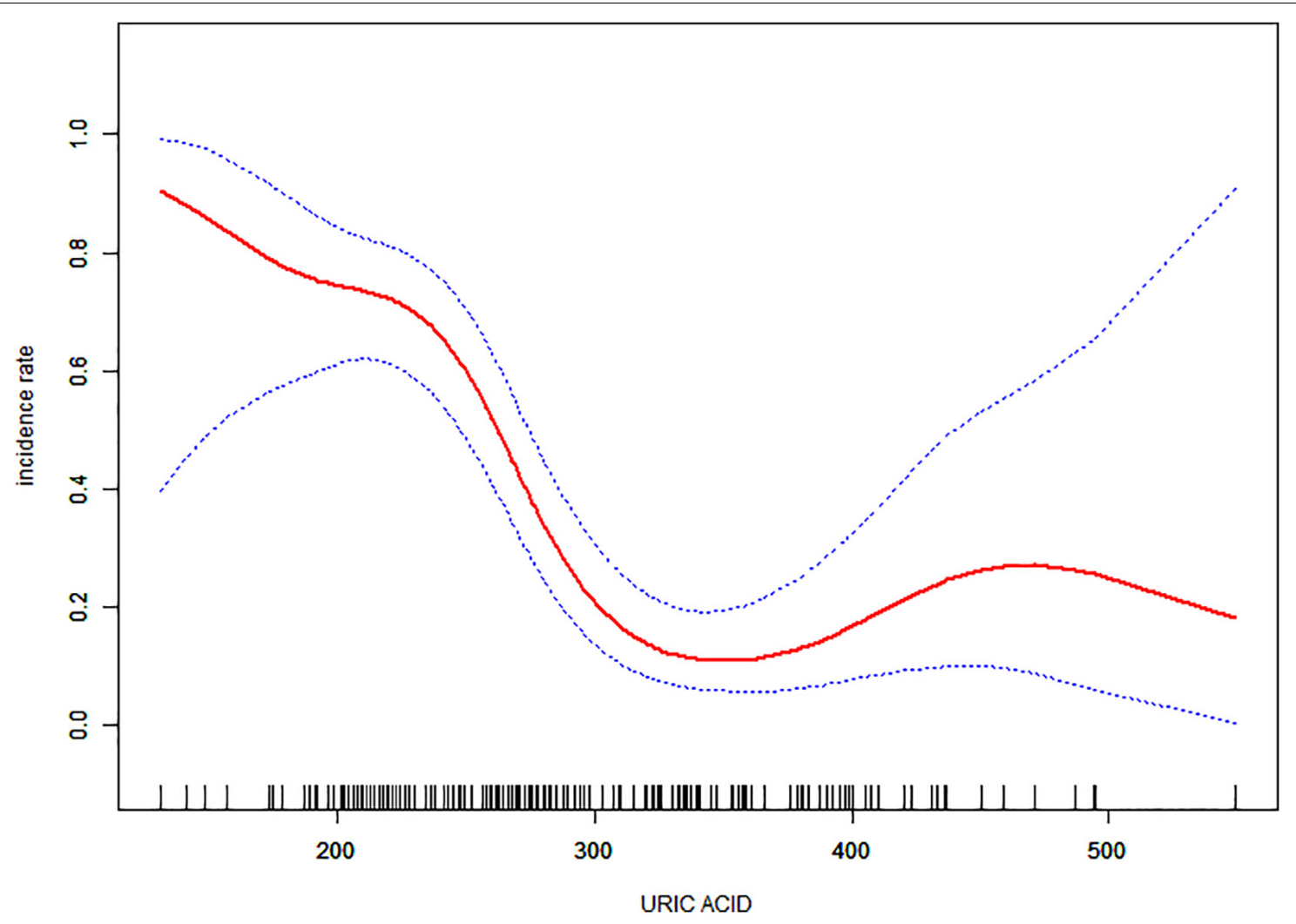

FIGURE 2 | The association of serum uric acid levels between the incidence rate of Meige's syndrome. The adjusted data for the incidence rate of Meige's syndrome is plotted against serum uric acid levels with a curve indicating the shaped relationship between the two. A threshold serum uric acid level of $337 \mu \mathrm{mol} / \mathrm{L}$ existed for the regulation of uric acid.

TABLE 5 | The threshold effect of serum uric acid levels on score of BFMDRS assessments.

\begin{tabular}{lcc}
\hline & OR (95\% Cl) & $\boldsymbol{P}$-value \\
\hline Uric acid $<330 \mu \mathrm{mol} / \mathrm{L}$ & $-0.065(-0.075,-0.056)$ & $<0.001$ \\
Uric acid $\geq 330 \mu \mathrm{mol} / \mathrm{L}$ & $0.006(-0.010,0.021)$ & 0.186 \\
\hline
\end{tabular}

Adjusted for age, gender, history of hypertension. Cl, confidence interval; $O R$, odds ratio.

shown in Figure 1. Our results indicated that the URIC values performed the best in diagnosis, with an AUC value of 0.781 and the specificity and sensitivity were 0.7368 and 0.7875 , respectively (Figure 1).

Non-linear relationships between serum URIC and the incidence rate of MS and the BFMDRS score were detected. The incidence rate of MS increased as serum URIC levels decreased to the turning point (URIC $=337 \mu \mathrm{mol} / \mathrm{L})$. With a URIC level $\geq 337 \mu \mathrm{mol} / \mathrm{L}$, the predicted dose-response curve conformed to the horizontal line (Table 4 and Figure 2). Similarly, the severity of symptoms increased with decreasing URIC levels to the turning point (URIC $=330 \mu \mathrm{mol} / \mathrm{L})$. Likewise, the OR of the incidence rate of $\mathrm{MS}$ was 0.975 (95\% CI: $0.968-0.983$ ), and -0.065 (95\% CI: -0.075 to -0.056 ) for BFMDRS scores (Table 5 and Figure 3).

\section{DISCUSSION}

Numerous studies have been conducted on the relationship between serum URIC levels and neurological diseases. Nonetheless, the association is still controversial because of the small sample sizes; besides, other confounders such as sex and age may have a certain impact. Currently, there are disputes regarding the role of URIC content in the prognosis prediction of neurological disorders. Increased URIC content has been suggested as a protective factor for functional results (Xue et al., 2017), whereas other studies suggest URIC content as a risk factor (Jung et al., 2018). The effect of URIC levels on MS has not been investigated until now.

This study analyzed URIC contents in MS patients and normal subjects. The results suggested that MS patients had decreased serum URIC content compared with normal subjects, suggesting that decreased URIC levels might serve as an MSrelated risk factor. After adjusting for sex, age, and hypertension history, the heterogeneity remained significant. This result conforms to the opinion that URIC shows neuroprotection, which may be related to its role as an iron chelator and antioxidant (Davies et al., 1986). OS is related to numerous central nervous system (CNS) diseases, such as the dopaminergic cell decomposition of $\mathrm{PD}$ and early neuronal characteristics 


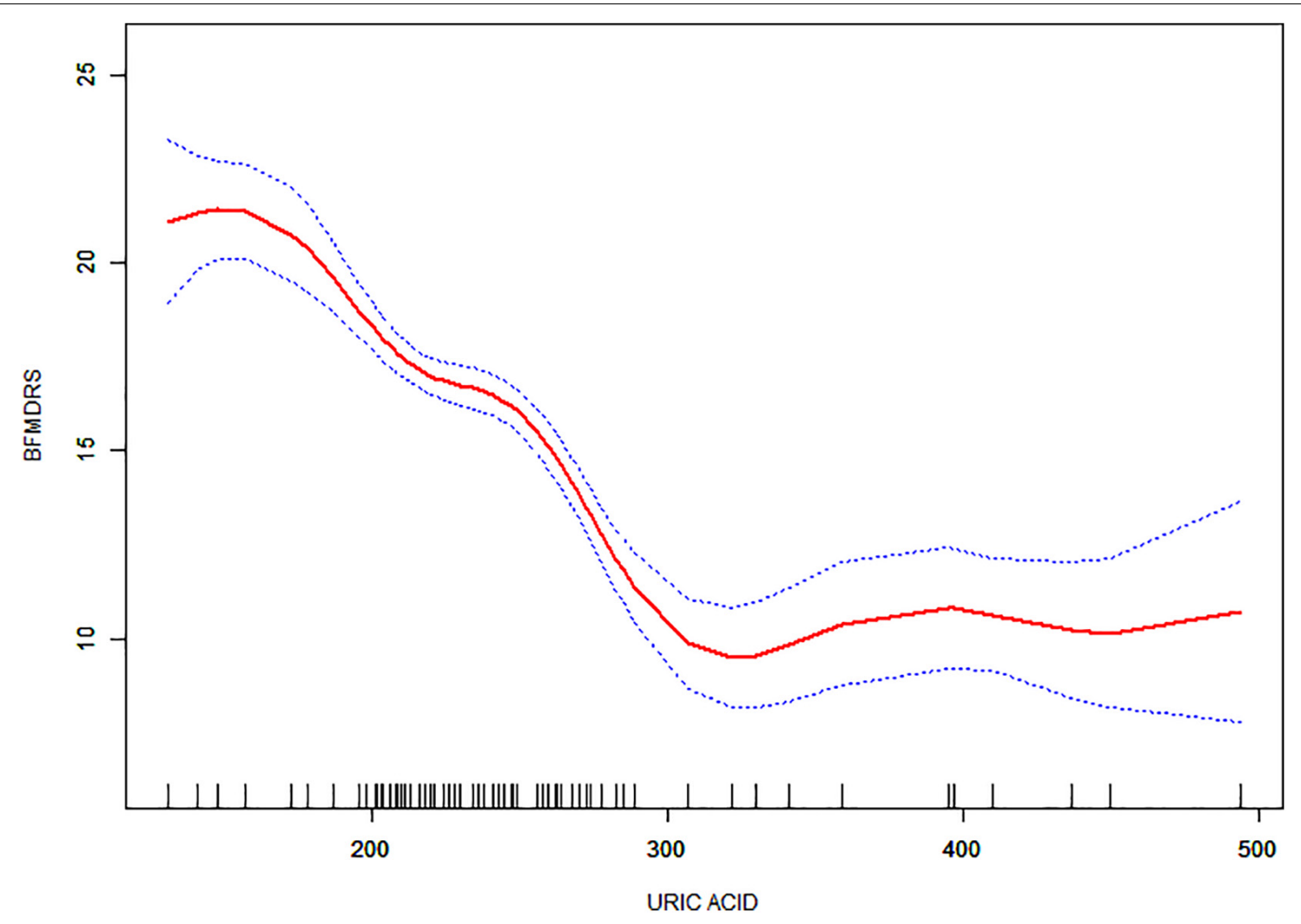

FIGURE 3 | The association between serum uric acid levels and Burke-Fahn-Marsden dystonia rating scale scores. A non-linear relationship was observed, and a threshold serum uric acid of $330 \mu \mathrm{mol} / \mathrm{L}$ existed for the regulation of uric acid.

in Alzheimer's disease (Moreira et al., 2006; Wei et al., 2018). Moreover, free radicals such as peroxynitrite may facilitate axonal demyelination and inflammation (Hooper et al., 2000). Therefore, the prevention of OS can delay the occurrence of such CNS diseases and improve their prognostic outcomes. URIC is a potent endogenous antioxidant that functions in resisting OS-mediated neuronal death and neurodegeneration, as suggested by in vivo and in vitro studies (Chen et al., 2013; Bartoli et al., 2017). As a result, the present work indicates that URIC exerts antioxidant and neuroprotective effects in MS.

URIC contents in MS cases are similar to those in PD cases, indicating a similar pathophysiological mechanism underlying neuronal injury. The neuroprotective effects of glutathione have been demonstrated in several studies (Fitzmaurice et al., 2003). The reduced glutathione content in PD is possibly due to the aberrant production, utilization, and catabolism of URIC and/or additional CNS antioxidants (Albers et al., 1999). Therefore, the decreased glutathione content in MS may be due to the decreased URIC content. Further studies are needed to explore this relationship.

Our study also found an interesting non-linear relationship between URIC levels, MS incidence and symptom severity, indicating that the incidence and severity of MS increased significantly when URIC was lower than 337 and $330 \mu \mathrm{mol} / \mathrm{L}$. This suggests that monitoring and regulating URIC may be important for both patients with MS and healthy individuals. It is well known that the normal value of URIC is 149$416 \mu \mathrm{mol} / \mathrm{L}$ in males and $89-357 \mu \mathrm{mol} / \mathrm{L}$ in females (Maiuolo et al., 2016). When URIC is higher than normal, there is a risk of gout and cardiovascular disease (Jung et al., 2018). However, this study demonstrates that low serum URIC levels increase the risk of MS and the severity of symptoms in patients with MS. In summary, it is necessary to control serum URIC at an appropriate level. Therefore, our results suggest that controlling serum uric acid levels in the range of $337 \mu \mathrm{mol} / \mathrm{L}$ to the upper normal value is a reasonable goal. Thus, serum uric acid levels are a double-edged sword, and regulating URIC levels at an appropriate level is beneficial for preventing and delaying neurological diseases.

This study has some limitations. First, the MS cohort had a small sample size. Second, this study only extracted data regarding whether the study subjects had hypertension, but no information on detailed medication was gathered. Third, this study did not determine the long-term effects of URIC on MS.

The present study was the first to analyze the association of serum URIC levels with MS. Low serum URIC levels predict 
a higher MS risk and serious clinical symptoms. This study sheds light on future avenues of research for the prevention and treatment of MS.

\section{DATA AVAILABILITY STATEMENT}

The raw data supporting the conclusions of this article will be made available by the authors, without undue reservation.

\section{ETHICS STATEMENT}

The studies involving human participants were reviewed and approved by Shanghai General Hospital Institutional Ethics

\section{REFERENCES}

Albers, D. S., Augood, S. J., Martin, D. M., Standaert, D. G., Vonsattel, J. P., and Beal, M. F. (1999). Evidence for oxidative stress in the subthalamic nucleus in progressive supranuclear palsy. J. Neurochem. 73, 881-884. doi: 10.1046/j.14714159.1999.0730881.x

Ascherio, A., LeWitt, P. A., Xu, K., Eberly, S., Watts, A., Matson, W. R., et al. (2009). Urate as a predictor of the rate of clinical decline in Parkinson disease. Arch. Neurol. 66, 1460-1468. doi: 10.1001/archneurol.200 9.247

Bartoli, F., Carra, G., and Clerici, M. (2017). Purinergic dysfunction in bipolar disorder: any role for the antioxidant uric acid as a trait and state biomarker? Psychiatry Clin. Neurosci. 71:417. doi: 10.1111/pcn.1 2518

Becker, B. F., Reinholz, N., Leipert, B., Raschke, P., Permanetter, B., and Gerlach, E. (1991). Role of uric acid as an endogenous radical scavenger and antioxidant. Chest 100, 176S-181S. doi: 10.1378/chest.100.3_Supplement.176S

Chen, X., Burdett, T. C., Desjardins, C. A., Logan, R., Cipriani, S., Xu, Y., et al. (2013). Disrupted and transgenic urate oxidase alter urate and dopaminergic neurodegeneration. Proc. Natl. Acad. Sci. U. S. A. 110, 300-305. doi: 10.1073/pnas. 1217296110

Church, W. H., and Ward, V. L. (1994). Uric acid is reduced in the substantia nigra in Parkinson's disease: effect on dopamine oxidation. Brain Res. Bull. 33, 419-425. doi: 10.1016/0361-9230(94)90 285-2

Davies, K. J., Sevanian, A., Muakkassah-Kelly, S. F., and Hochstein, P. (1986). Uric acide iron ion complexes. A new aspect of the antioxidant functions of uric acid. Biochem. J. 235, 747-754. doi: 10.1042/bj235 0747

Davis, J. W., Grandinetti, A., Waslien, C. I., Ross, G. W., White, L. R., and Morens, D. M. (1996). Observations on serum uric acid levels and the risk of idiopathic Parkinson's disease. Am. J. Epidemiol. 144, 480-484. doi: 10.1093/oxfordjournals.aje.a008954

Defazio, G., Abbruzzese, G., Livrea, P., and Berardelli, A. (2004). Epidemiology of primary dystonia. Lancet Neurol. 3, 673-678. doi: 10.1016/S1474-4422(04) 00907-X

Fitzmaurice, P. S., Ang, L., Guttman, M., Rajput, A. H., Furukawa, Y., and Kish, S. J. (2003). Nigral glutathione deficiency is not specific for idiopathic Parkinson's disease. Mov. Disord. 18, 969-976. doi: 10.1002/mds.1 0486

Gao, X., O’Reilly, E. J., Schwarzschild, M. A., and Ascherio, A. (2016). Prospective study of plasma urate and risk of Parkinson disease in men and women. Neurology 86, 520-526. doi: 10.1212/WNL.000000000000 2351

Greene, P., Kang, U. J., and Fahn, S. (1995). Spread of symptoms in idiopathic torsion dystonia. Mov. Disord. 10, 143-152. doi: 10.1002/mds.87010 0204
Committee Second People's Hospital of Hefei Institutional Ethics Committee. The patients/participants provided their written informed consent to participate in this study.

\section{AUTHOR CONTRIBUTIONS}

HG and ZG jointly completed the experiment and the writing. $\mathrm{BC}$ and WY took overall control of the whole study. All authors contributed to the article and approved the submitted version.

\section{ACKNOWLEDGMENTS}

We would like to thank Editage for English language editing.

Hooper, D. C., Scott, G. S., Zborek, A., Mikheeva, T., Kean, R. B., Koprowski, H., et al. (2000). Uric acid, a peroxynitrite scavenger, inhibits CNS inflammation, blood-CNS barrier permeability changes, and tissue damage in a mouse model of multiple sclerosis. FASEB J. 14, 691-698. doi: 10.1096/fasebj.14. 5.691

Jesus, S., Perez, I., Caceres-Redondo, M. T., Carrillo, F., Carballo, M., GomezGarre, P., et al. (2013). Low serum uric acid concentration in Parkinson's disease in southern Spain. Eur. J. Neurol. 20, 208-210. doi: 10.1111/j.14681331.2012.03745.x

Jung, J. Y., Choi, Y., Suh, C. H., Yoon, D., and Kim, H. (2018). Effect of fenofibrate on uric acid level in patients with gout. Sci. Rep. 8:16767. doi: 10.1038/s41598018-35175-Z

LeDoux, M. S. (2009). Meige syndrome: what's in a name? Parkinsonism Relat. Disord. 15, 483-489. doi: 10.1016/j.parkreldis.2009.0 4.006

Maiuolo, J., Oppedisano, F., Gratteri, S., Muscoli, C., and Mollace, V. (2016). Regulation of uric acid metabolism and excretion. Int. J. Cardiol. 15, 8-14. doi: 10.1016/j.ijcard.2015.08.109

McFarland, N. R., Burdett, T., Desjardins, C. A., Frosch, M. P., and Schwarzschild, M. A. (2013). Postmortem brain levels of urate and precursors in Parkinson's disease and related disorders. Neurodegener Dis. 12, 189-198. doi: 10.1159/ 000346370

Moreira, P. I., Honda, K., Zhu, X., Nunomura, A., Casadesus, G., Smith, M. A., et al. (2006). Brain and brawn Parallels in oxidative strength. Neurology 66, 97-101. doi: 10.1212/01.wnl.0000192307.1510 3.83

Pandey, S., and Sharma, S. (2017). Meige's syndrome: history, epidemiology, clinical features, pathogenesis and treatment. J. Neurol. Sci. 372, 162-170. doi: 10.1016/j.jns.2016.11.053

Poewe, W., Seppi, K., Tanner, T. M., Halliday, G. M., Brundin, P., Volkmann, J., et al. (2017). Parkinson disease. Nat. Rev. Dis. Primer 3, 1-21. doi: 10.1038/ nrdp. 2017.13

Savitt, J. M., Dawson, V. L., and Dawson, T. M. (2006). Diagnosis and treatment of Parkinson disease: molecules to medicine. J. Clin. Invest. 116, 1744-1754. doi: 10.1172/JCI29178

Steele, J. C., Richardson, J. C., and Olszewski, J. (2014). Progressive supranuclear palsy: a heterogeneous degeneration involving the brain stem, basal ganglia and cerebellum with vertical gaze and pseudobulbar palsy, nuchal dystonia and dementia. Semin. Neurol. 34, 129-150. doi: 10.1055/s-0034-137 7058

Stocker, R., Yamamoto, Y., McDonagh, A. F., Glazer, A. N., and Ames, B. N. (1987). Bilirubin is an antioxidant of possible physiological importance. Science 235, 1043-1046. doi: 10.1126/science.302 9864

Wei, Z., Li, X., Li, X., Liu, Q., and Cheng, Y. (2018). Oxidative stress in Parkinson's disease: a systematic review and meta-analysis. Front. Mol. Neurosci. 11:236 doi: 10.3389/fnmol.2018.00236 
Wen, M., Zhou, B., Chen, Y. H., Ma, Z. L., Gou, Y., Zhang, C. L., et al. (2017). Serum uric acid levels in patients with Parkinson's disease: a meta-analysis. PLoS One 12:e0173731. doi: 10.1371/journal.pone.017 3731

Xue, L., Liu, Y., Xue, H., Xue, J., Sun, K., Wu, L., et al. (2017). Low uric acid is a risk factor in mild cognitive impairment. Neuropsychiatr. Dis. Treat. 13, 2363-2367. doi: 10.2147/NDT.S14 5812

Conflict of Interest: The authors declare that the research was conducted in the absence of any commercial or financial relationships that could be construed as a potential conflict of interest.
Publisher's Note: All claims expressed in this article are solely those of the authors and do not necessarily represent those of their affiliated organizations, or those of the publisher, the editors and the reviewers. Any product that may be evaluated in this article, or claim that may be made by its manufacturer, is not guaranteed or endorsed by the publisher.

Copyright (c) 2021 Guan, Geng, Yuan and Chang. This is an open-access article distributed under the terms of the Creative Commons Attribution License (CC BY). The use, distribution or reproduction in other forums is permitted, provided the original author(s) and the copyright owner(s) are credited and that the original publication in this journal is cited, in accordance with accepted academic practice. No use, distribution or reproduction is permitted which does not comply with these terms. 